\title{
Solving Differential Equations with Z-numbers by utilizing Fuzzy Sumudu Transform
}

\author{
Raheleh Jafari \\ Department of Information and Communication Technology \\ Agder University College, 4876 \\ Grimstad, Norway \\ Email: jafari3339@yahoo.com
}

\author{
Sina Razvarz \\ Departamento de Control Automático \\ CINVESTAV-IPN (National Polytechnic Institute) \\ Mexico City, Mexico \\ Email:srazvarz@yahoo.com
}

\author{
Alexander Gegov \\ School of Computing, University of Portsmouth \\ Buckingham Building, Portsmouth PO1 3HE, UK \\ Email: alexander.gegov@port.ac.uk
}

\begin{abstract}
The uncertain nonlinear systems can be modeled with fuzzy differential equations (FDEs) and the solutions of these equations are applied to analyze many engineering problems. However, it is very difficult to obtain solutions of FDEs. In this paper, the solutions of FDEs are approximated by utilizing the fuzzy Sumudu transform (FST) method. Here, the uncertainties are in the sense of $Z$-numbers. Important theorems are laid down to illustrate the properties of FST. The theoretical analysis and simulation results show that this new technique is effective to estimate the solutions of FDEs.
\end{abstract}

Keywords-fuzzy Sumudu transform; fuzzy differential equation; Z-number

\section{INTRODUCTION}

In many physical and dynamical processes, mathematical modeling leads to the deterministic initial and boundary value problems. In practice, the boundary values may be different from crisp and displays in the form of unknown parameters [36]. When the parameters or the states of the differential equations are uncertain, they can be modeled with FDE. In recent years, many methods have used FDE for modeling and control of uncertain nonlinear systems [14], [15], [16], [17], [18], [20]. The basic idea of the fuzzy derivative was first introduced in [10]. Then it is extended in [11]. The first-order fuzzy initial value problem, as well as fuzzy partial differential equation, have been studied in [25]. By generalizing the differentiability, [6] gave an analytical solution. The Lipschitz condition, as well as the theorem for existence and uniqueness of the solution related to FDEs, are discussed in [4], [33]. In [5], the analytical solutions of second order FDE are obtained. The analytical solutions of third order linear FDE are found in [13]. By the interval-valued method, [30] examined the basic solutions of nonlinear FDEs with generalized differentiability.

A novel technique in order to solve FDEs is laid down based on the Sumudu transform. Sumudu transform along with broad applications has been utilized in the area of system engineering and applied physics [9], [26], [29]. In [8], some simple and deeper fundamental theorems and properties of the Sumudu Transform were generalized. In [24], Sumudu transform is applied to the system of differential equations. In [1], Sumudu transform is used in order to find the solution of fuzzy partial differential equations. In [23], Sumudu transform has been used to solve fractional differential equations.

In this paper, we use FST to approximate the Z-number solutions of the FDEs. The Z-number is a new concept that is subjected to a higher potential to demonstrate the information of the human being as well as to utilize in information processing [35]. Z-numbers can be regarded as to answer questions and carry out the decisions [21]. There exist very few structure based on the theoretical concept of Z-numbers [12]. [2] gave an inception, which results in the extension of the Z-numbers. [22] generated a theorem to convert the Znumbers to the usual fuzzy sets.

In this work, the FST reduces the FDE to an algebraic equation. A very important property of the FST is that it can solve the equation without resorting to a new frequency domain. The procedure of switching FDEs to an algebraic equation is cited in [4] and is stated as an operational calculus. This paper is one of the first attempts in finding the solutions of FDEs based on Z-numbers using FST. In section II and section III, some preliminary definitions along with properties related to FST which are useful throughout this paper are demonstrated. In section IV, solving FDEs based on Z-numbers using the methodology of FST has been discussed. In section $\mathrm{V}$, a benchmark example along with comparisons is utilized in order to demonstrate the effectiveness of our proposed method.

\section{PRELIMINARIES}

Prior to the introduction of the FST, some concepts related to the Z-numbers are laid down in this section [7], [19], [28].

Definition 1: A Z-number has two components $Z=$ $[B(a), \tilde{p}]$. The primary component $B(a)$ is restriction on a real-valued uncertain variable $a$. The secondary component $\tilde{p}$ is a measure of reliability of $B . \tilde{p}$ can be reliability, strength of belief, probability or possibility. When $B(a)$ is a fuzzy number and $\tilde{p}$ is the probability distribution of $a$, the Z-number is stated as $Z^{+}$-number. When $B(a)$ as well as $\tilde{p}$ are fuzzy numbers, the $\mathrm{Z}$-number is stated as $Z^{-}$-number.

The $Z^{+}$-number carries more information when compared with $Z^{-}$-number. In this paper, we utilize the definition of 
$Z^{+}$-number, i.e., $Z=[B, \tilde{p}], B$ is a fuzzy number and $\tilde{p}$ is a probability distribution.

To express the fuzzy number the most common membership functions are utilized in this paper. The popular membership functions are the triangular function

$$
\mu_{B}=F\left(\lambda_{1}, \lambda_{2}, \lambda_{3}\right)= \begin{cases}\frac{a-\lambda_{1}}{\lambda_{2}-\lambda_{1}} & \lambda_{1} \leq a \leq \lambda_{2} \\ \frac{\lambda_{3}-\zeta}{\lambda_{3}-\lambda_{2}} & \lambda_{2} \leq a \leq \lambda_{3}\end{cases}
$$

otherwise $\mu_{B}=0$, and trapezoidal function

$$
\mu_{B}=F\left(\lambda_{1}, \lambda_{2}, \lambda_{3}, \lambda_{4}\right)=\left\{\begin{array}{cc}
\frac{a-\lambda_{1}}{\lambda_{2}-\lambda_{1}} & \lambda_{1} \leq a \leq \lambda_{2} \\
\frac{\lambda_{4}-a}{\lambda_{4}-\lambda_{3}} & \lambda_{3} \leq a \leq \lambda_{4} \\
1 & \lambda_{2} \leq a \leq \lambda_{3}
\end{array}\right.
$$

otherwise $\mu_{B}=0$.

The probability measure is defined as

$$
\tilde{P}=\int_{R} \mu_{B}(a) \tilde{p}(a) d a
$$

where $\tilde{p}$ is the probability density of $a$, also $R$ is the restriction on $\tilde{p}$. For discrete Z-numbers

$$
\tilde{P}(B)=\sum_{i=1}^{n} \mu_{B}\left(a_{i}\right) \tilde{p}\left(a_{i}\right)
$$

Definition 2: The $r$-level of the Z-number $Z=(B, \tilde{P})$ is illustrated as

$$
[Z]^{r}=\left([B]^{r},[\tilde{p}]^{r}\right)
$$

where $0<r \leq 1$. $[\tilde{p}]^{r}$ is computed by the Nguyen's theorem

$$
[\tilde{p}]^{r}=\tilde{p}\left([B]^{r}\right)=\tilde{p}\left(\left[\underline{B}^{r}, \bar{B}^{r}\right]\right)=\left[\underline{\tilde{P}}^{r}, \overline{\tilde{P}}^{r}\right]
$$

where $\tilde{p}\left([B]^{r}\right)=\left\{\tilde{p}(a) \mid a \in[B]^{r}\right\}$. So $[Z]^{r}$ can be demonstrated as the form $r$-level of a fuzzy number

$$
[Z]^{r}=\left(\underline{Z}^{r}, \bar{Z}^{r}\right)=\left(\left(\underline{B}^{r}, \underline{\tilde{P}}^{r}\right),\left(\bar{B}^{r}, \overline{\tilde{P}}^{r}\right)\right)
$$

where $\underline{\tilde{P}}^{r}=\underline{B}^{r} \tilde{p}\left({\underline{a_{i}}}^{r}\right), \overline{\tilde{P}}^{r}=\bar{B}^{r} \tilde{p}\left({\overline{a_{i}}}^{r}\right),\left[a_{i}\right]^{r}=\left({\underline{a_{i}}}^{r},{\overline{a_{i}}}^{r}\right)$.

Similar to the fuzzy numbers, the Z-numbers are also incorporated with three elementary operations; $\oplus, \ominus$, and $\odot$ which are named as addition, subtraction, and multiplication. The operations in this paper are different definitions with [34]. The $r$-level of Z-numbers is applied to simplify the operations.

Suppose $Z_{1}=\left(B_{1}, \tilde{p}_{1}\right)$ and $Z_{2}=\left(B_{2}, \tilde{p}_{2}\right)$ be two discrete Z-numbers expressing the uncertain variables $a_{1}$ and $a_{2}, \sum_{\iota=1}^{n} \tilde{p}_{1}\left(a_{1 \iota}\right)=1, \sum_{\iota=1}^{n} \tilde{p}_{2}\left(a_{2 \iota}\right)=1$. The operations are displayed as

$$
Z_{12}=Z_{1} * Z_{2}=\left(B_{1} * B_{2}, \tilde{p}_{1} * \tilde{p}_{2}\right)
$$

where $* \in\{\oplus, \ominus, \odot\}$.

The operations for the fuzzy numbers are illustrated as [15]

$$
\begin{gathered}
{\left[B_{1} \oplus B_{2}\right]^{r}=\left[B_{1}\right]^{r}+\left[B_{2}\right]^{r}=\left[\underline{B}_{1}^{r}+\underline{B}_{2}^{r}, \bar{B}_{1}^{r}+\bar{B}_{2}^{r}\right]} \\
{\left[B_{1} \ominus B_{2}\right]^{r}=\left[B_{1}\right]^{r}-\left[B_{2}\right]^{r}=\left[\underline{B}_{1}^{r}-\underline{B}_{2}^{r}, \bar{B}_{1}^{r}-\bar{B}_{2}^{r}\right]} \\
{\left[B_{1} \odot B_{2}\right]^{r}=\left(\begin{array}{c}
\min \left\{\underline{B}_{1}^{r} \underline{B}_{2}^{r}, \underline{B}_{1}^{r} \bar{B}_{2}^{r}, \bar{B}_{1}^{r} \underline{B}_{2}^{r}, \bar{B}_{1}^{r} \bar{B}_{2}^{r}\right\} \\
\max \left\{\underline{B}_{1}^{r} \underline{B}_{2}^{r}, \underline{B}_{1}^{r} \bar{B}_{2}^{r}, \bar{B}_{1}^{r} \underline{B}_{2}^{r}, \bar{B}_{1}^{r} \bar{B}_{2}^{r}\right\}
\end{array}\right)}
\end{gathered}
$$

For all $\tilde{p}_{1} * \tilde{p}_{2}$ operations, we use convolutions for the discrete probability distributions

$$
\tilde{p}_{1} * \tilde{p}_{2}=\sum_{i} \tilde{p}_{1}\left(a_{1, i}\right) \tilde{p}_{2}\left(a_{2,(n-i)}\right)=\tilde{p}_{12}(a)
$$

The above definitions satisfy the Hukuhara difference [3],

$$
\begin{gathered}
Z_{1} \ominus_{H} Z_{2}=Z_{12} \\
Z_{1}=Z_{2} \oplus Z_{12}
\end{gathered}
$$

If $Z_{1} \ominus_{H} Z_{2}$ prevails, the $r$-level is

$$
\left[Z_{1} \ominus_{H} Z_{2}\right]^{r}=\left[\underline{Z}_{1}^{r}-\underline{Z}_{2}^{r}, \bar{Z}_{1}^{r}-\bar{Z}_{2}^{r}\right]
$$

Obviously, $Z_{1} \ominus_{H} Z_{1}=0, Z_{1} \ominus Z_{1} \neq 0$.

Also the above definitions satisfy the generalized Hukuhara difference [7]

$$
Z_{1} \ominus_{g H} Z_{2}=Z_{12} \Longleftrightarrow\left\{\begin{array}{l}
\text { 1) } Z_{1}=Z_{2} \oplus Z_{12} \\
\text { 2) } Z_{2}=Z_{1} \oplus(-1) Z_{12}
\end{array}\right.
$$

It is easy to display that 1) and 2) in combination are genuine if and only if $Z_{12}$ is a crisp number. With respect to $r$-level we have $\left[Z_{1} \ominus_{g H} Z_{2}\right]^{r}=\left[\min \left\{\underline{Z}_{1}^{r}-\underline{Z}_{2}^{r}, \bar{Z}_{1}^{r}-\bar{Z}_{2}^{r}\right\}, \max \left\{\underline{Z}_{1}^{r}-\right.\right.$ $\left.\left.\underline{Z}_{2}^{r}, \bar{Z}_{1}^{r}-\bar{Z}_{2}^{r}\right\}\right]$ and If $Z_{1} \ominus_{g H} Z_{2}$ and $Z_{1} \ominus_{H} Z_{2}$ subsist, $Z_{1} \ominus_{H}$ $Z_{2}=Z_{1} \ominus_{g H} Z_{2}$. The conditions for the existence of $Z_{12}=$ $Z_{1} \ominus_{g H} Z_{2} \in E$ are

$$
\begin{gathered}
\text { 1) }\left\{\begin{array}{c}
\underline{Z}_{12}^{r}=\underline{Z}_{1}^{r}-\underline{Z}_{2}^{r} \text { and } \bar{Z}_{12}^{r}=\bar{Z}_{1}^{r}-\bar{Z}_{2}^{r} \\
\text { with } \underline{Z}_{12}^{r} \text { increasing, } \bar{Z}_{12}^{r} \text { decreasing, } \underline{Z}_{12}^{r} \leq \bar{Z}_{12}^{r}
\end{array}\right. \\
\text { 2) }\left\{\begin{array}{c}
\underline{Z}_{12}^{r}=\bar{Z}_{1}^{r}-\bar{Z}_{2}^{r} \text { and } \bar{Z}_{12}^{r}=\underline{Z}_{1}^{r}-\underline{Z}_{2}^{r} \\
\text { with } \underline{Z}_{12}^{r} \text { increasing, } \bar{Z}_{12}^{r} \text { decreasing, } \underline{Z}_{12}^{r} \leq \bar{Z}_{12}^{r}
\end{array}\right.
\end{gathered}
$$

where $\forall r \in[0,1]$

Definition 3: Suppose $\hat{Z}$ demonstrates the space of $Z$ numbers, then the $r$-level of $Z$-number valued function $\Psi$ : $\left[a_{1}, a_{2}\right] \rightarrow \hat{Z}$ is defined as

$$
\Psi(\phi, r)=[\underline{\Psi}(\phi, r), \bar{\Psi}(\phi, r)]
$$

where $\phi \in \hat{Z}$, and $r \in[0,1]$.

Based on the definition of Generalized Hukuhara difference, the $\mathrm{gH}$-derivative of $\Psi$ at $\phi_{0}$ is defined as

$$
\Psi^{\prime}\left(\phi_{0}\right)=\lim _{h \rightarrow 0} \frac{1}{h}\left[\Psi\left(\phi_{0}+h\right) \ominus_{g H} \Psi\left(\phi_{0}\right)\right]
$$

In (17), $\Psi\left(\phi_{0}+h\right)$ and $\Psi\left(\phi_{0}\right)$ represents similar style with $Z_{1}$ and $Z_{2}$ respectively defined in (15).

By implementing the $r$-level (5) to initial value problem, $\phi^{\prime}(t)=\psi(t, \phi(t))$, we generate two Z-number valued functions: $\underline{\psi}[t, \underline{\phi}(a, r), \bar{\phi}(a, r)]$ and $\bar{\psi}[t, \underline{\phi}(a, r), \bar{\phi}(a, r)]$.

The initial value problem can be equivalent to the following relation

$$
\begin{array}{r}
i)\left\{\begin{array}{l}
\phi^{\prime}=\psi[t, \phi(a, r), \bar{\phi}(a, r)] \\
\overline{\overline{\phi^{\prime}}}=\overline{\bar{\psi}}[t, \bar{\phi}(a, r), \bar{\phi}(a, r)]
\end{array}\right. \\
i i)\left\{\begin{array}{l}
\phi^{\prime}=\bar{\psi}[t, \underline{\phi}(a, r), \bar{\phi}(a, r)] \\
\overline{\overline{\phi^{\prime}}}=\underline{\psi}[t, \underline{\phi}(a, r), \bar{\phi}(a, r)]
\end{array}\right.
\end{array}
$$

Definition 4: The function $\psi:\left[a_{1}, a_{2}\right] \longrightarrow \hat{Z}$ is integrable on $\left[a_{1}, a_{2}\right]$, if it satisfies in the below mentioned relation

$$
\int_{a_{1}}^{\infty} \psi(x) d x=\left(\int_{a_{1}}^{\infty} \underline{\psi}(x, r) d x, \int_{a_{1}}^{\infty} \bar{\psi}(x, r) d x\right)
$$


If $\psi(x)$ be a Z-number valued function, as well as $q(x)$ be a Z-number Riemann integrable on $\left[a_{1}, \infty\right]$ then $\psi(x) \oplus q(x)$ can be a Z-number Riemann integrable on $\left[a_{1}, \infty\right]$. Therefore,

$$
\int_{a_{1}}^{\infty}(\psi(x) \oplus q(x)) d x=\int_{a}^{\infty} \psi(x) d x \oplus \int_{a}^{\infty} q(x) d x
$$

\section{FUZZY SUMUdU TRANSFORM}

Fuzzy initial and boundary value problems can be resolved by utilizing fuzzy Laplace transform [4]. In this paper, the FST methodology for Z-number is illustrated, furthermore the properties of this methodology is stated. By applying the FST methodology, the FDE based on Z-numbers is reduced to an algebraic equation. The main advantageous of the FST is that it can resolve the equation without resorting to a new frequency domain. The methodology of converting FDEs to an algebraic equation is expressed in [4].

Definition 5: Suppose $\psi(t)$ be a continuous Z-number valued function, also, $\psi(\hat{B} t) \odot e^{-t}$ be an improper Z-number Riemann integrable on $[0, \infty)$, where $\hat{B} \in \hat{Z}$. Accordingly, $\int_{0}^{\infty} \psi(\hat{B} t) \odot e^{-t} d t$ is expressed as FST and it is defined by $\Omega(\hat{B})=\mathbf{S}[\psi(t)]=\int_{0}^{\infty} \psi(\hat{B} t) \odot e^{-t} d t$, where $0 \leq \hat{B}<K$, $K \in \hat{Z}$, also $e^{-t}$ is real valued function. Based on the Theorem 2 we have the following relation

$\int_{0}^{\infty} \psi(\hat{B} t) \odot e^{-t} d t=\left(\int_{0}^{\infty} \underline{\psi}(\hat{B} t, r) e^{-t} d t, \int_{0}^{\infty} \bar{\psi}(\hat{B} t, r) e^{-t} d t\right)$

Let

$$
\begin{aligned}
& \mathbf{S}[\psi(t, r)]=\int_{0}^{\infty} \psi(\hat{\bar{B}} t, r) e^{-t} d t \\
& \mathbf{S}[\overline{\bar{\psi}}(t, r)]=\int_{0}^{\infty} \overline{\bar{\psi}}(\hat{B} t, r) e^{-t} d t
\end{aligned}
$$

hence we obtain the following relation

$$
\mathbf{S}[\psi(t)]=(\mathbf{S}[\underline{\psi}(t, r), \mathbf{S} \bar{\psi}(t, r)])
$$

Theorem 1: Suppose $\psi^{\prime}(t)$ be a Z-number value integrable function, as well as $\psi(t)$ be the primitive of $\psi^{\prime}(t)$ on $[0, \infty)$. Therefore,

$$
\mathbf{S}\left[\psi^{\prime}(t)\right]=\frac{1}{\hat{B}} \odot \mathbf{S}[\psi(t)] \ominus\left(\frac{1}{\hat{B}} \odot[\psi(0)]\right)
$$

where $\psi$ is considered to be (i)-differentiable, or

$$
\mathbf{S}\left[\psi^{\prime}(t)\right]=\frac{-1}{\hat{B}} \odot[\psi(0)] \ominus\left(\frac{-1}{\hat{B}} \odot \mathbf{S}[\psi(t)]\right)
$$

where $\psi$ is considered to be (ii)-differentiable.

Proof. For arbitrary fixed $r \in[0,1]$ we have

$$
\begin{aligned}
& \frac{1}{\hat{B}} \odot \mathbf{S}[\psi(t)] \ominus\left(\frac{1}{\hat{B}} \odot \psi(0)\right) \\
& =\left(\frac{1}{\hat{B}} \mathbf{S}[\underline{\psi}(t, r)]-\frac{1}{\hat{B}} \mathbf{S}[\underline{\psi}(0, r)], \frac{1}{\hat{B}} \mathbf{S}[\bar{\psi}(t, r)]-\frac{1}{\hat{B}} \mathbf{S}[\bar{\psi}(0, r)]\right)
\end{aligned}
$$

We have the following relations

$$
\begin{aligned}
& \mathbf{S}\left[\bar{\psi}^{\prime}(t, r)\right]=\frac{1}{\hat{B}} \mathbf{S}[\bar{\psi}(t, r)]-\frac{1}{\hat{\hat{B}}}[\bar{\psi}(0, r)] \\
& \mathbf{S}\left[\underline{\psi}^{\prime}(t, r)\right]=\frac{1}{\hat{B}} \mathbf{S}[\underline{\psi}(t, r)]-\frac{1}{\hat{B}}[\underline{\psi}(0, r)]
\end{aligned}
$$

Hence, we obtain

$$
\frac{1}{\hat{B}} \odot \mathbf{S}[\psi(t)] \ominus\left(\frac{1}{\hat{B}} \odot \psi(0)\right)=\left(\mathbf{S}\left[\underline{\psi}^{\prime}(t, r)\right], \mathbf{S}\left[\bar{\psi}^{\prime}(t, r)\right]\right)
$$

If $\psi$ is cosidered to be (i)-differentiable, so

$$
\frac{1}{\hat{B}} \odot \mathbf{S}[\psi(t)] \ominus\left(\frac{1}{\hat{B}} \odot \psi(0)\right)=\mathbf{S}\left[\psi^{\prime}(t)\right]
$$

Let $\psi$ is (ii)-differentiable. For arbitrary fixed $r \in[0,1]$ we obtain

$$
\begin{aligned}
& \frac{-1}{\hat{B}} \odot[\psi(0)] \ominus\left(\frac{-1}{\hat{B}} \odot \mathbf{S}[\psi(t)]\right) \\
& =\left(\frac{-1}{\hat{B}} \bar{\psi}(0, r)+\frac{1}{\hat{B}} \mathbf{S}[\bar{\psi}(t, r)], \frac{-1}{\hat{B}} \underline{\psi}(0, r)+\frac{1}{\hat{B}} \mathbf{S}[\underline{\psi}(t, r)]\right)
\end{aligned}
$$

The above equation can be written as the following relation

$$
\begin{aligned}
& \frac{-1}{\hat{B}} \odot[\psi(0)] \ominus\left(\frac{-1}{\hat{B}} \odot \mathbf{S}[\psi(t)]\right) \\
& =\left(\frac{1}{\hat{B}} \mathbf{S}[\bar{\psi}(t, r)]-\frac{1}{\hat{B}} \bar{\psi}(0, r), \frac{1}{\hat{B}} \mathbf{S}[\underline{\psi}(t, r)]-\frac{1}{\hat{B}} \underline{\psi}(0, r)\right)
\end{aligned}
$$

We obtain

$$
\begin{aligned}
& \mathbf{S}\left[\bar{\psi}^{\prime}(t, r)\right]=\frac{1}{\hat{\hat{B}}} \mathbf{S}[\bar{\psi}(t, r)]-\frac{1}{\hat{\hat{\beta}}} \bar{\psi}(0, r) \\
& \mathbf{S}\left[\underline{\psi}^{\prime}(t, r)\right]=\frac{1}{\hat{B}} \mathbf{S}[\underline{\psi}(t, r)]-\frac{1}{\hat{B}} \underline{\psi}(0, r)
\end{aligned}
$$

So, we have

$$
\left(\frac{-1}{\hat{B}} \psi(0)\right) \ominus\left(\frac{-1}{\hat{B}} \odot \mathbf{S}[\psi(t)]\right)=\left(\mathbf{S}\left[\bar{\psi}^{\prime}(t, r)\right], \mathbf{S}\left[\underline{\psi^{\prime}}(t, r)\right]\right)
$$

Hence

$$
\left(\frac{-1}{\hat{B}} \psi(0)\right) \ominus\left(\frac{-1}{\hat{B}} \odot \mathbf{S}[\psi(t)]\right)=\mathbf{S}\left(\left[\bar{\psi}^{\prime}(t, r)\right],\left[\underline{\psi}^{\prime}(t, r)\right]\right)
$$

Since $\psi$ is (ii)-differentiable, therefore,

$$
\left(\frac{-1}{\hat{B}} \psi(0)\right) \ominus\left(\frac{-1}{\hat{B}} \odot \mathbf{S}[\psi(t)]\right)=\mathbf{S}\left[\psi^{\prime}(t)\right]
$$

Theorem 2: Taking into consideration that Sumudu transform is a linear transformation, so if $\psi(t)$ and $\vartheta(t)$ be continuous Z-number valued functions, moreover $k_{1}$ as well as $k_{2}$ be constant, therefore the following relation can be obtained

$\mathbf{S}\left[\left(k_{1} \odot \psi(t)\right) \oplus\left(k_{2} \odot \vartheta(t)\right)\right]=\left(k_{1} \odot \mathbf{S}[\psi(t)]\right) \oplus\left(k_{2} \odot \mathbf{S}[\vartheta(t)]\right)$

Proof. We have

$$
\begin{aligned}
& \mathbf{S}\left[\left(k_{1} \odot \psi(t)\right) \oplus\left(k_{2} \odot \vartheta(t)\right)\right] \\
& =\int_{0}^{\infty}\left(k_{1} \odot \varphi(\hat{B} t) \oplus k_{2} \odot \vartheta(\hat{B} t)\right) \odot e^{-t} d t \\
& =\int_{0}^{\infty} k_{1} \odot \psi(\hat{B} t) \odot e^{-t} d t \oplus \int_{0}^{\infty} k_{2} \odot \vartheta(\hat{B} t) \odot e^{-t} d t \\
& =k_{1} \odot\left(\int_{0}^{\infty} \psi(\hat{B} t) \odot e^{-t} d t\right) \oplus k_{2} \odot\left(\int_{0}^{\infty} \vartheta(\hat{B} t) \odot e^{-t} d t\right) \\
& =k_{1} \odot \mathbf{S}[\psi(t)] \oplus k_{2} \odot \mathbf{S}[\vartheta(t)]
\end{aligned}
$$

Therefore, we conclude

$\mathbf{S}\left[\left(k_{1} \odot \psi(t)\right) \oplus\left(k_{2} \odot \vartheta(t)\right)\right]=\left(k_{1} \odot \mathbf{S}[\psi(t)]\right) \oplus\left(k_{2} \odot \mathbf{S}[\vartheta(t)]\right)$

Lemma 1: Assume that the $\psi(t)$ is a continuous Z-number valued function on $[0, \infty)$, also $\gamma \geq 0$, thus

$$
\mathbf{S}[\gamma \odot \psi(t)]=\gamma \odot \mathbf{S}[\psi(t)]
$$

Proof. Fuzzy Sumudu transform $\gamma \odot \psi(t)$ is defined as

$$
\mathbf{S}[\gamma \odot \psi(t)]=\int_{0}^{\infty} \gamma \odot \psi(\hat{B} t) \odot e^{-t} d t
$$

furthermore, we have

$$
\int_{0}^{\infty} \gamma \odot \psi(\hat{B} t) \odot e^{-t} d t=\gamma \odot \int_{0}^{\infty} \psi(\hat{B} t) \odot e^{-t} d t
$$

therefore,

$$
\mathbf{S}[\gamma \odot \psi(t)]=\gamma \odot \mathbf{S}[\psi(t)]
$$




\section{SOLVING FUZZY INITIAL VALUE PROBLEM WITH FUZZY SUMUDU TRANSFORM METHOD}

Consider the following fuzzy initial value problem based on Z-numbers

$$
\left\{\begin{aligned}
\phi^{\prime}(t) & =\psi(t, \phi(t)), \\
\phi(0) & =(\underline{\phi}(0, r), \bar{\phi}(0, r)), \quad 0<r \leq 1
\end{aligned}\right.
$$

where $\psi(t, \phi(t))$ is a Z-number function. The Z-number function $\psi(t, \phi(t))$ is the mapping of $\psi: R \times \hat{Z} \rightarrow \hat{Z}$. By utilizing FST method for Z-numbers, we obtain

$$
\mathbf{S}\left[\phi^{\prime}(t)\right]=\mathbf{S}[\psi(t, \phi(t))]
$$

The resolving process of Eq. (44) is base on the following cases.

Case 1: Assume that the $\phi^{\prime}(t)$ is (i)-differentiable. Base on the Theorem 2 we extract

$$
\begin{aligned}
& \phi^{\prime}(t)=\left(\phi^{\prime}(t, r), \bar{\phi}^{\prime}(t, r)\right) \\
& \mathbf{S}\left[\phi^{\prime}(t)\right]=\left(\frac{1}{\hat{B}} \odot \mathbf{S}[\phi(t)]\right) \ominus \frac{1}{\hat{B}} \phi(0)
\end{aligned}
$$

Eq. (45) can be displayed as following relation

$$
\left\{\begin{array}{l}
\mathbf{S}[\psi(t, \phi(t), r)]=\frac{1}{\hat{B}} \mathbf{S}[\phi(t, r)]-\frac{1}{\hat{B}} \phi(0, \alpha) \\
\mathbf{S}[\overline{\bar{\psi}}(t, \phi(t), r)]=\frac{1}{\hat{B}} \mathbf{S}[\overline{\bar{\phi}}(t, r)]-\frac{1}{\hat{B}}(0, r)
\end{array}\right.
$$

where

$$
\left\{\begin{aligned}
\psi & (t, \phi(t), r)=\min \{\psi(t, \hat{B}) \mid \hat{B} \in(\underline{\phi}(t, r), \bar{\phi}(t, r))\} \\
\overline{\bar{\psi}}(t, \phi(t), r) & =\max \{\psi(t, \hat{B}) \mid \hat{B} \in(\underline{\phi}(t, r), \bar{\phi}(t, r))\}
\end{aligned}\right.
$$

Accordingly, Eq. (47) can be resolved on the basis of the following assumptions

$$
\begin{aligned}
& \mathbf{S}[\phi(t, r)]=U_{1}(\hat{B}, r) \\
& \mathbf{S}[\overline{\bar{\phi}}(t, r)]=U_{2}(\hat{B}, r)
\end{aligned}
$$

where $U_{1}(\hat{B}, r)$, as well as $U_{2}(\hat{B}, r)$ are the Z-number solutions of the Eq. (47). By applying inverse Sumudu transform, $\underline{\phi}(t, r)$ as well as $\bar{\phi}(t, r)$ are computed as

$$
\begin{aligned}
\phi & (t, r)=\mathbf{S}^{-1}\left[U_{1}(\hat{B}, r)\right] \\
\bar{\phi}(t, r) & =\mathbf{S}^{-1}\left[U_{2}(\hat{B}, r)\right]
\end{aligned}
$$

Case 2: Assume that the $\phi^{\prime}(t)$ is (ii)-differentiable. Based on the Theorem 2 we extract

$$
\begin{aligned}
& \phi^{\prime}(t)=\left(\bar{\phi}^{\prime}(t, r), \phi^{\prime}(t, r)\right) \\
& \mathbf{S}\left[\phi^{\prime}(t)\right]=\left(\frac{-1}{\hat{B}} \odot \phi(0)\right) \ominus\left(\frac{-1}{\hat{B}} \odot \mathbf{S}[\phi(t)]\right)
\end{aligned}
$$

Eq. (50) can be displayed as following relation

$$
\left\{\begin{array}{l}
\mathbf{S}[\psi(t, \phi(t), r)]=\frac{1}{\hat{B}} \mathbf{S}[\phi(t, r)]-\frac{1}{\hat{B}} \phi(0, r) \\
\mathbf{S}[\overline{\bar{\psi}}(t, \phi(t), r)]=\frac{1}{\hat{B}} \mathbf{S}[\overline{\bar{\phi}}(t, r)]-\frac{1}{\hat{B}}(0, r)
\end{array}\right.
$$

where

$$
\left\{\begin{array}{l}
\psi(t, \phi(t), r)=\min \{\psi(t, \hat{B}) \mid \hat{B} \in(\underline{\phi}(t, r), \bar{\phi}(t, r))\} \\
\overline{\bar{\psi}}(t, \phi(t), r)=\max \{\psi(t, \hat{B}) \mid \hat{B} \in(\underline{\phi}(t, r), \bar{\phi}(t, r))\}
\end{array}\right.
$$

Accordingly, Eq. (52) can be resolved on the basis of the following assumptions

$$
\begin{aligned}
& \mathbf{S}\left(\phi(t, r)=V_{1}(\hat{B}, r)\right. \\
& \mathbf{S}\left(\overline{\bar{\phi}}(t, r)=V_{2}(\hat{B}, r)\right.
\end{aligned}
$$

where $V_{1}(\hat{B}, r)$, and $V_{2}(\hat{B}, r)$ are the Z-number solutions of the Eq. (52). By applying inverse Sumudu transform, $\phi(t, r)$ and $\bar{\phi}(t, r)$ are computed as

$$
\begin{aligned}
\phi(t, r) & =\mathbf{S}^{-1}\left[V_{1}(\hat{B}, r)\right] \\
\overline{\bar{\phi}}(t, r) & =\mathbf{S}^{-1}\left[V_{2}(\hat{B}, r)\right]
\end{aligned}
$$

\section{APPLICATION}

In this section, a real example is used to demonstrate how to apply FST method in order to find the solution of FDEs on the basis of $\mathrm{Z}$-numbers.

Example 1 A tank with a heating system is displayed in Figure 1 , where $\tilde{R}=0.5$, the thermal capacitance is $\tilde{C}=2$ also the temperature is $\psi$. The model is formulated as follows[4], [27],

$$
\left\{\begin{array}{l}
\phi^{\prime}(t)=-\frac{1}{\tilde{R} \tilde{C}} \phi(t), \quad 0 \leq t \leq T \\
\phi(0)=[(\underline{\phi}(0, r), \bar{\phi}(0, r)), p(0.8,0.9,1)]
\end{array}\right.
$$

By utilizing the FST method based on Z-number we obtain

$$
\begin{aligned}
& \mathbf{S}\left[\phi^{\prime}(t)\right]=\mathbf{S}[-\phi(t)] \\
& \mathbf{S}\left[\phi^{\prime}(t)\right]=\int_{0}^{\infty} \phi^{\prime}(\hat{B} t) \odot e^{-t} d t
\end{aligned}
$$

where $0 \leq \hat{B}<K$. By considering case 1 for Z-numbers the following relation is obtained

$$
\mathbf{S}\left[\phi^{\prime}(t)\right]=\frac{1}{\hat{B}} \odot(\mathbf{S}[\phi(t)] \ominus \phi(0))=\frac{1}{\hat{B}} \mathbf{S}[\phi(t)] \ominus \frac{1}{\hat{B}} \phi(0)
$$

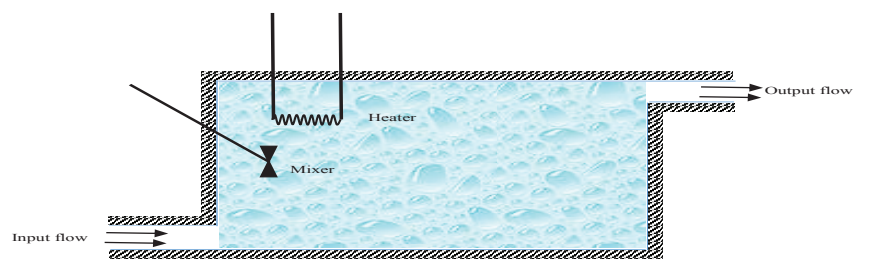

Fig. 1. A tank with a heating system

Therefore

$$
-\mathbf{S}[\phi(t)]=\frac{1}{\hat{B}} \mathbf{S}[\phi(t)] \ominus \frac{1}{\hat{B}} \phi(0)
$$

Based on the Eq. (46), we have

$$
\left\{\begin{aligned}
-\mathbf{S}[\bar{\phi}(t, r)] & =\frac{1}{\hat{B}} \mathbf{S}[\phi(t, r)]-\frac{1}{\hat{B}} \phi(0, r) \\
-\mathbf{S}[\underline{\phi}(t, r)] & =\frac{1}{\hat{B}} \mathbf{S}[\overline{\bar{\phi}}(t, r)]-\frac{1}{\hat{\phi}}(0, r)
\end{aligned}\right.
$$

Therefore, the Z-number solution of Eq. (59) is extracted as $[(\mathbf{S}[\bar{\phi}(t, r)], \mathbf{S}[\phi(t, r)]), p(0.8,0.94,1)]$ where

$$
\left\{\begin{array}{l}
\mathbf{S}[\bar{\phi}(t, r)]=\left(\frac{-1}{\hat{B}^{2}-1}\right) \bar{\phi}(0, r)+\left(\frac{\hat{B}}{\hat{B}^{2}-1}\right) \underline{\phi}(0, r) \\
\mathbf{S}[\underline{\phi}(t, r)]=\left(\frac{-1}{\hat{B}^{2}-1}\right) \underline{\phi}(t, r)+\left(\frac{\hat{B}}{\hat{B}^{2}-1}\right) \bar{\phi}(0, r)
\end{array}\right.
$$

By utilizing the inverse Sumudu transform for Z-numbers, we have

$$
\left\{\begin{array}{l}
\mathbf{S}[\bar{\phi}(t, r)]=\bar{\phi}(0, r) \mathbf{S}^{-1}\left(\frac{-1}{\hat{B}^{2}-1}\right)+\underline{\phi}(0, r) \mathbf{S}^{-1}\left(\frac{\hat{B}}{\hat{B}^{2}-1}\right) \\
\mathbf{S}[\underline{\phi}(t, r)]=\underline{\phi}(0, r) \mathbf{S}^{-1}\left(\frac{-1}{\hat{B}^{2}-1}\right)+\bar{\phi}(0, r) \mathbf{S}^{-1}\left(\frac{\hat{B}}{\hat{B}^{2}-1}\right)
\end{array}\right.
$$


where

$$
\left\{\begin{array}{l}
\bar{\phi}(t, r)=e^{t}\left(\frac{\bar{\phi}(0, r)-\underline{\phi}(0, r)}{2}\right)+e^{-t}\left(\frac{\bar{\phi}(0, r)+\underline{\phi}(0, r)}{2}\right) \\
\underline{\phi}(t, r)=e^{t}\left(\frac{\underline{\phi}(0, r)-\bar{\phi}(0, r)}{2}\right)+e^{-t}\left(\frac{\underline{\phi}(0, r)+\bar{\phi}(0, r)}{2}\right)
\end{array}\right.
$$

By considering case 2 for Z-numbers the following relation is obtained

$$
\mathbf{S}\left[\phi^{\prime}(t)\right]=\left(\frac{-1}{\hat{B}} \mathbf{S}[\phi(t)]\right) \ominus\left(\frac{-1}{\hat{B}} \phi(0)\right)
$$

Hence

$$
-\mathbf{S}[\phi(t)]=\left(\frac{-1}{\hat{B}} \mathbf{S}[\phi(t)]\right) \ominus\left(\frac{-1}{\hat{B}} \phi(0)\right)
$$

Based on the above relations, Eq. (55) is illustrated as

$$
\left\{\begin{aligned}
-\mathbf{S}[\phi(t, r)] & =\frac{1}{\hat{B}} \mathbf{S}[\phi(t, r)]-\frac{1}{\hat{B}} \phi(0, r) \\
-\mathbf{S}[\overline{\bar{\phi}}(t, r)] & =\frac{1}{\hat{B}} \mathbf{S}[\overline{\bar{\phi}}(t, r)]-\frac{1}{\hat{B}}(0, r)
\end{aligned}\right.
$$

So, the Z-number solution of Eq. (65) is displayed as $[(\mathbf{S}[\underline{\phi}(t, r)], \mathbf{S}[\bar{\phi}(t, r)]), p(0.8,0.9,1)]$ where

$$
\left\{\begin{array}{l}
\mathbf{S}[\phi(t, r)]=\phi(0, r)\left(\frac{1}{\hat{B}+1}\right) \\
\mathbf{S}[\bar{\phi}(t, r)]=\overline{\bar{\phi}}(t, r)\left(\frac{1}{\hat{B}+1}\right)
\end{array}\right.
$$

By utilizing the inverse Sumudu transform for Z-numbers, we have

$$
\left\{\begin{aligned}
\phi(t, r) & =\phi(0, r) \mathbf{S}^{-1}\left(\frac{1}{\hat{B}+1}\right) \\
\overline{\bar{\phi}}(t, r) & =\overline{\bar{\phi}}(0, r) \mathbf{S}^{-1}\left(\frac{1}{\hat{B}+1}\right)
\end{aligned}\right.
$$

where

$$
\left\{\begin{array}{l}
\phi(t, r)=e^{-t} \phi(0, r) \\
\overline{\bar{\phi}}(t, r)=e^{-t} \overline{\bar{\phi}}(0, r)
\end{array}\right.
$$

If the initial condition is taken to be a symmetric triangular Z-number as $\phi(0)=[(-a(1-r), a(1-r)), p(0.8,0.9,1)]$, so

Case 1 :

$$
\left\{\begin{array}{c}
\phi(t, r)=e^{t}(-a(1-r)) \\
\bar{\phi}(t, r)=e^{t}(a(1-r))
\end{array}\right.
$$

Case 2:

$$
\left\{\begin{array}{l}
\phi(t, r)=e^{-t}(-a(1-r)) \\
\bar{\phi}(t, r)=e^{-t}(a(1-r))
\end{array}\right.
$$

Approximation errors based on Z-numbers are shown in Table 1. These errors are the differences between the exact and the approximation solutions, for two different methods: FST and Average Euler method [32].

Table 1. Approximation errors based on Z-numbers

\begin{tabular}{|c|l|l|}
\hline$\alpha$ & \multicolumn{1}{|c|}{ FST } & \multicolumn{1}{|c|}{ Average Euler } \\
\hline 0 & {$[(0.0078,0.0195), \mathrm{p}(0.8,0.86,0.94)]$} & {$[(0.0138,0.0215), \mathrm{p}(0.7,0.8,0.87)]$} \\
\hline 0.2 & {$[(0.0085,0.0169), \mathrm{p}(0.75,0.8,0.9)]$} & {$[(0.0188,0.0286), \mathrm{p}(0.7,0.8,0.87)]$} \\
\hline 0.6 & {$[(0.0058,0.0115), \mathrm{p}(0.8,0.9,1)]$} & {$[(0.0182,0.0198), \mathrm{p}(0.7,0.8,0.92)]$} \\
\hline 0.8 & {$[(0.0091,0.0123), \mathrm{p}(0.7,0.75,0.8)]$} & {$[(0.0148,0.0189), \mathrm{p}(0.6,0.7,0.8)]$} \\
\hline 1 & {$[(0.0132,0.0132), \mathrm{p}(0.7,0.8,0.9)]$} & {$[(0.0710,0.0710), \mathrm{p}(0.6,0.75,0.87)]$} \\
\hline
\end{tabular}

The following formula is utilized to transfer the Z-numbers to fuzzy numbers,

$$
\sigma=\frac{\int \phi \pi_{P}(\phi) d \phi}{\int \pi_{P}(\phi) d \phi}
$$

By taking in to consideration $Z$ $[(0.0078,0.0195), p(0.8,0.86,0.94)]$,
$Z^{\sigma}=[0.0078,0.0195 ; 0.86]$, accordingly $Z^{\prime}=$

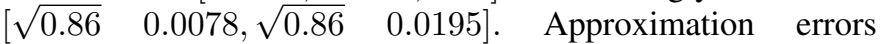
based on fuzzy numbers are shown in Table 2 .

Table 2. Approximation errors based on fuzzy numbers

\begin{tabular}{|c|c|c|}
\hline$\alpha$ & FST & Average Euler \\
\hline 0 & {$[0.0072,0.0180]$} & {$[0.0123,0.0192]$} \\
\hline 0.2 & {$[0.0076,0.0151]$} & {$[0.0168,0.0255]$} \\
\hline 0.6 & {$[0.0055,0.0109]$} & {$[0.0162,0.0177]$} \\
\hline 0.8 & {$[0.0078,0.0106]$} & {$[0.0123,0.0158]$} \\
\hline 1 & {$[0.0118,0.0118]$} & {$[0.0614,0.0614]$} \\
\hline
\end{tabular}

Figure 2 shows the corresponding error plots based on fuzzy numbers. FST is more accurate than the Average Euler method. Figure 3 and Figure 4 demonstrate the corresponding solution plots based on fuzzy numbers.

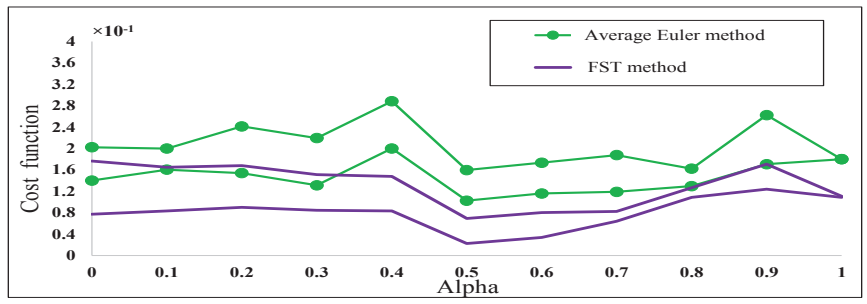

Fig. 2. The lower and upper bounds of absolute errors based on fuzzy numbers

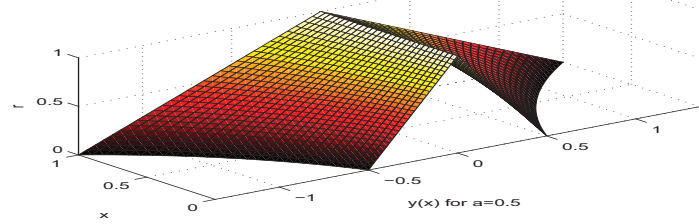

Fig. 3. The solution of FDE under case 1 consideration based on fuzzy numbers

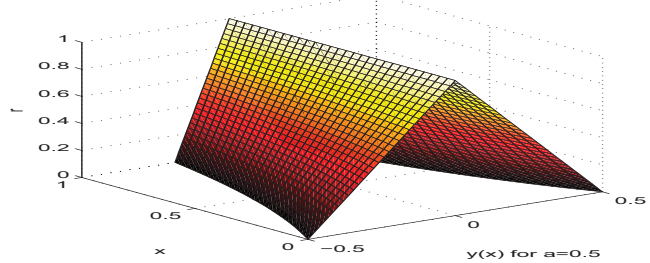

Fig. 4. The solution of FDE under case 2 consideration based on fuzzy numbers

By implementing Z-numbers the degree of reliability of the information can be increased. The comparison between the Znumber $Z=[(0.0078,0.0195), p(0.8,0.86,0.94)]$ and fuzzy number $[0.0072,0.0180]$ is displayed in Figure 5 . It can be seen that the Z-number incorporates with various information, also the solution related to the Z-number is more precise. The membership function regarding the restriction in the Znumber is considered to be $\mu_{B_{Z}}=[0.0078,0.0195]$. It can be in probability form. 


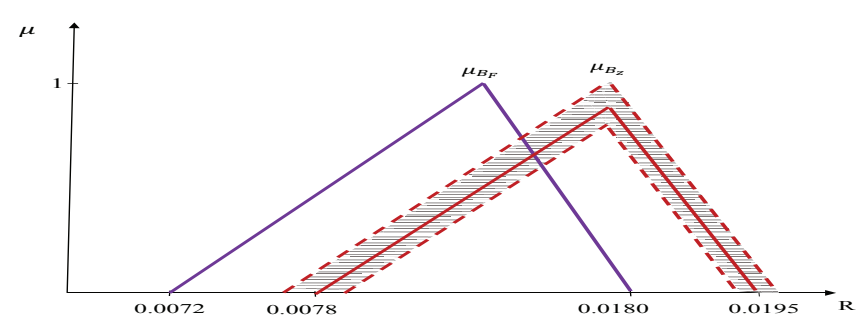

Fig. 5. The comparison between the Z-number and fuzzy number

\section{CONCLUSION}

In this paper, a novel method based on the FST is proposed in order to find the solution of the first order FDEs on the basis of Z-numbers. The new method is clarified by utilizing the concept of strongly generalized differentiability. By using the FST method, the FDE converts to an algebraic problem. Some essential theorems are laid down in order to demonstrate the properties of the FST. A real example is applied to demonstrate the effectiveness of the proposed technique. This work has a significant contribution in initializing a superior starting point for such extensions. The future work is the application of the mentioned methodology for fuzzy partial differential equations on the basis of Z-numbers.

\section{REFERENCES}

[1] N.A. Abdul Rahman, M.Z. Ahmad, Fuzzy Sumudu transform for solving fuzzy partial differential equations, J. Nonlinear Sci. Appl. Vol.9, pp.3226-3239, 2016

[2] R.A. Aliev, A.V. Alizadeh, O.H. Huseynov, The arithmetic of discrete Z-numbers, Inform. Sci. Vol.290, pp.134155, 2015.

[3] R.A. Aliev, W. Pedryczb, V. Kreinovich, O.H. Huseynov, The general theory of decisions, Inform. Sci. Vol.327, pp.125-148, 2016.

[4] T. Allahviranloo, M.B. Ahmadi, Fuzzy Laplace Transform, Soft Computing. Vol.14, pp.235-243, 2010.

[5] T. Allahviranloo, N.A. Kiani, M. Barkhor- dari, Toward the exiistence and uniqueness of solution of second-order fuzzy differential equations, Inform. Sci. Vol.179, pp.1207-1215, 2009.

[6] B. Bede, I.J. Rudas, A.L. Bencsik, First order linear fuzzy differential equations under generalized differentiability, Inform. Sci. Vol.177, pp.1648-1662, 2007.

[7] B. Bede, L. Stefanini, Generalized differentiability of fuzzy-valued functions, Fuzzy Set Syst. Vol.230, pp.119-141, 2013.

[8] F.B.M. Belgacem, A.A. Karaballi, Sumudu transform fundamental properties investigations and applications, J. Appl. Math. Stoch. Anal. Vol.2006, doi. 10.1155/JAMSA/2006/91083, 2006.

[9] F.B.M. Belgacem, A.A. Karaballi, S.L. Kalla, Analytical investigations of the Sumudu transform and applications to integral production equations, Math. Prob. Eng. Vol.103, pp.103-118, 2003.

[10] S.L. Chang, L.A. Zadeh, On fuzzy mapping and control, IEEE Trans. Syst. Man Cybern. Vol.2, pp.30-34, 1972.

[11] D. Dubois, H. Prade, Towards fuzzy differential calculus: part 3 differentiation, Fuzzy Sets Syst. Vol.8, pp.225-233, 1982.

[12] L.A. Gardashova, Application of operational approaches to solving decision making problem using Z-Numbers, Journal of Applied Mathematics, Vol.5 pp.1323-1334, 2014.

[13] F. Hawrra, K.H. Amal, On fuzzy Laplace transform for fuzzy differential equations of the third order, Journal of Kerbala University, Vol.11, pp.251-256, 2013.

[14] R. Jafari, W. Yu, Artificial neural network approach for solving strongly degenerate parabolic and burgers-fisher equations, 12th International Conference on Electrical Engineering, Computing Science and Automatic Control, doi:10.1109/ICEEE.2015.7357914, 2015.
[15] R. Jafari, W. Yu, Fuzzy Control for Uncertainty Nonlinear Systems with Dual Fuzzy Equations, Journal of Intelligent and Fuzzy Systems. Vol.29, pp.1229-1240, 2015.

[16] R. Jafari, W. Yu, Uncertain nonlinear system control with fuzzy differential equations and Z-numbers, 18th IEEE International Conference on Industrial Technology, Canada, pp.890 -895, doi:10.1109/ICIT.2017.7915477, 2017.

[17] R. Jafari, W. Yu, Fuzzy Differential Equation for Nonlinear System Modeling with Bernstein Neural Networks, IEEE Access. doi:10.1109/ACCESS.2017.2647920, 2017.

[18] R. Jafari, W. Yu, Uncertainty Nonlinear Systems Modeling with Fuzzy Equations, Mathematical problems in Engineering. Vol.2017, doi:10.1155/2017/8594738, 2017.

[19] R. Jafari, W. Yu, X. Li, Numerical Solution of Fuzzy Equations with Z-numbers using Neural Networks, Intelligent Automation and Soft Computing, doi: 10.1080/10798587.2017.1327154, 2017

[20] R. Jafari, W. Yu, X. Li, S. Razvarz, Numerical Solution of Fuzzy Differential Equations with Z-numbers Using Bernstein Neural Networks, International Journal of Computa-tional Intelligence Systems, Vol.10, pp.12261237, 2017.

[21] B. Kang, D. Wei, Y. Li, Y. Deng, A method of converting Z-number to classical fuzzy number, Journal of Information and Computational Science, Vol.9, pp.703-709, 2012.

[22] B. Kang, D. Wei, Y. Li, Y. Deng, Decision making using Z-Numbers under uncertain environemnt, Journal of Computational Information Systems, Vol.8, pp.2807-2814, 2012.

[23] Q.D. Katatbeh, F.B.M. Belgacem, Applications of the Sumudu transform to fractional differential equations, Nonlinear Studies. Vol.18, pp.99-112, 2011

[24] A. Kiliman, H. Eltayeb, R.P. Agarwal, On Sumudu Transform and System of Differential Equations, Abstract and Applied Analysis, Vol.2010, Article ID 598702, 11 pages, 2010. doi:10.1155/2010/598702.

[25] P. Kloeden, Remarks on Peano-like theorems for fuzzy differential equations, Fuzzy Set Syst. Vol.44, pp.161-164, 1991.

[26] Y. Liu, W. Chen, A new iterational method for ordinary equations using Sumudu transform, Advances in Analysis, Vol.1, 2016.

[27] R.H. Pletcher, J.C. Tannehill, D. Anderson, Computational Fluid Mechanics and Heat Transfer, Taylor and Francis, 1997.

[28] S. Razvarz, M. Tahmasbi, Fuzzy equations and Z-numbers for nonlinear systems control, Procedia Computer Science, Vol.120, pp.923930, 2017.

[29] H.M. Srivastava, A. Khalili Golmankhaneh, D. Baleanu, X.J Yang, Local Fractional Sumudu Transform with Application to IVPs on Cantor Sets, Abstract and Applied Analysis. Article ID 620529, http://dx.doi.org/10.1155/2014/620529, 2014.

[30] L. Stefanini, B. Bede, Generalized Hukuhara differentiability of interval-valued functions and interval differential equations, J. Nonlinear Anal. (TMA). Vol.71, pp.1311-1328, 2009.

[31] V.L. Streeter, E.B. Wylie, K.W. Bedford, Fluid mechanics, New York: McGraw Hill, 1998

[32] S. Tapaswini, S. Chakraverty, Euler-based new solution method for fuzzy initial value problems, Int. J. Artificial. Intell. Soft. Comput. Vol.4, pp.58-79, 2014.

[33] V.A. Truong, V.H. Ngo, D.P. Nguyen, Global existence of solutions for interval-valued integro-differential equations under generalized $\mathrm{H}$ differentiability, Advances in Difference Equations. Vol.1, pp.217-233, 2013.

[34] L.A. Zadeh, Toward a generalized theory of uncertainty (GTU) an outline, Inform. Sci. vol.172, pp.1-40, 2005.

[35] L.A. Zadeh, Generalized theory of uncertainty (GTU)-principal concepts and ideas, Computational Statistics and Data Analysis, Vol.51, pp.15-46, 2006.

[36] D. Zwillinger, Handbook of differential equations, Gulf Professional Publishing. 1998. 